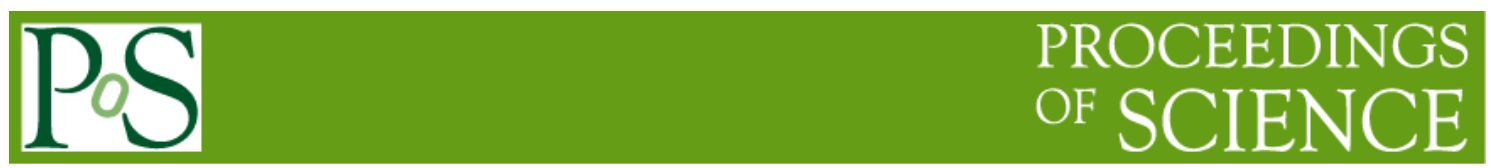

\title{
JIVE, what's next?
}

\section{Huib Jan van Langevelde ${ }^{1}$}

The Joint Institute for VLBI in Europe

Postbus 2, 7990 AA Dwingeloo, the Netherlands

And Sterrewacht Leiden, Leiden University

Postbus 9513, 2300 RA Leiden, the Netherlands

E-mail: langeveldeejive.nI

By the time of the $11^{\text {th }}$ EVN symposium in 2012, the European VLBI Network is a thriving scientific infrastructure with a significant user community and a healthy proposal pressure. It offers opportunities to address a breadth of important scientific topics, which feature in national and European astronomy roadmaps. Most of these science themes call for further enhancements of the sensitivity and image quality delivered by VLBI networks. At the heart of the EVN, JIVE is facilitating the central correlation operations, supporting the user community and monitoring the network performance. At the same time JIVE is pushing the technology for large capacity correlators that can connect VLBI networks with many elements in real-time. JIVE has been established as a Dutch foundation, operating on contributions from a number of EVN partners and with considerable support from the EC. In 2012 its achievements were evaluated in preparations for a new funding cycle. At this point the partners are considering changing the legal status of JIVE into a European Research Infrastructure Consortium.

11th European VLBI Network Symposium \& Users Meeting

October 9-12, 2012

Bordeaux, France

1

\section{Speaker}




\section{Introduction}

JIVE was established in 1993 to operate the 16-station Mk4 correlator. Over the years major technological advances were implemented, notably 10 years ago when hard-disk recording replaced the tape reels. Then VLBI underwent another revolution by providing realtime results, thereby enabling new science, boosting the reliability further and potentially enhancing the sensitivity. This has provided a natural growth path for this branch of highresolution radio astronomy. The increasing quality of the science return of VLBI has led to a solid user interest, addressing a wide variety of astronomical topics with the technique. With the introduction of portable, flexible software correlation, the role of JIVE is changing, with the emphasis on user services becoming even stronger. As the VLBI effort involves an increasing number of countries, for both telescopes and its user community, it is timely to consider how to proceed with this unique research infrastructure, made up of the EVN and JIVE.

\section{The EVN}

\subsection{Science topics}

The European VLBI Network is addressing an increasingly wide range of astronomical topics [13], starting from the classical area of jets in Active Galactic Nuclei (AGN, e.g. [9][2]) and the AGN/starburst connection in cosmological fields [9]. An important goal in this field is to probe jet physics close to the black hole origin. Major breakthroughs are expected in this research area with the deployment of sub-mm VLBI with ALMA, which promises to map the shadow of the central Galactic black hole, SgrA* [8]. In some radio-loud galaxies the signs of recent starburst can be detected directly by resolving individual supernova shells and remnants [14][1]. VLBI can contribute to the on-going debate on the relation between massive black hole and AGN evolution and the starburst galaxies phenomena.

Through the research of interstellar masers VLBI, can probe the detailed physics in a range of astrophysical targets. Extragalactic masers are often associated with either extreme starburst events or disks at the hearts of AGN, or both. Galactic masers allow one to zoom in on the detailed physics (kinematics, radiation fields and magnetic forces) in both evolved stars, as well as very young objects [5][16][17]. From the same maser observations usually also very fundamental distance measurements are obtained [15]. Some stellar parallaxes are also obtainable by continuum observations [3], both contributing to our understanding of the Galaxy itself.

The study of transient radio sources has flourished with the development of real-time VLBI, addressing GRB's at cosmic distances, as well as stellar scale events in our Galaxy [4][11]. But currently the samples under study are limited in size, until much better sensitivity becomes available. Together with large field-of-view survey instruments, like LOFAR [20] providing many more triggers, this can be expected to be a booming industry in a matter of years. Pulsar capabilities must feature prominently as well in the coming years, as VLBI pulsar astrometry will be in demand for population studies that harvest the results from a number of SKA pathfinders. Finally, it has been demonstrated that VLBI offers very interesting scientific 
applications for planetary Space missions, by providing accurate positions of spacecraft [6][12]. In fact the RadioAstron mission itself [7] may profit from being observed during observations, to pinpoint the space antenna for use in the primary science observations.

Addressing such a wide range of topics, it is clear that European VLBI offers an extremely versatile research facility. It can contribute to many of the themes that have featured in various roadmap exercises that are being conducted on European and national levels, from cosmology, galaxy evolution to the life cycle of stars and even the origin of planets and life.

\subsection{EVN development programme}

To be complementary to other, lower resolution, radio facilities, VLBI must continue to enhance its operational bandwidth. Improvements in digital equipment, but also in antenna receivers are necessary to reach the sensitivity levels that are relevant for modern radio studies. While the full 1024Mbps data-rate has been offered regularly for both disk-based and e-VLBI observations, the EVN has started to push the sensitivity by testing with 4 Gbps in 2012. A critical item has been the introduction of the new filters and digitizers, the DBBC system in the case of the EVN. This system is needed for any new telescope that joins the array and eventually for all stations to go beyond 1024Mbps. Moreover, the EVN bandwidth is limited by the inherent width of the IF systems of the telescopes, and this must be addressed at many stations at the same time. Eventually, there is the ambition to go to even higher bandwidths, which will be mostly useful at frequencies above $10 \mathrm{GHz}$. Here possibly up to $2 \mathrm{GHz}$ bandwidth could be realized in each polarization, calling for at least $16 \mathrm{Gbps}$ data-rates.

It is exciting to note that more antennas are becoming available for the EVN, often with impressive sensitivities. Adding many more antennas to the network also improves the visibility plane coverage and it has been demonstrated that arrays with more than 20 antennas can produce images with very impressive image fidelity, something that for example is required to derive mass distribution functions in gravitational lenses. Many projects in the EVN science areas will be stimulated by more coverage at higher frequencies, notably $22 \mathrm{GHz}$ and $43 \mathrm{GHz}$, for example in masers research. In particular for jet physics there is definitely also a science case for observing with intercontinental baselines at these frequencies.

The operational model of the EVN is evolving towards much more real-time operations. The objective of the current development programmes is to implement an e-VLBI component for every VLBI experiment that is carried out. By implementing transparent buffering mechanisms at telescope and correlator end, one can address all the current and future bottlenecks in e-VLBI. We envision an operational model in which all data that can be transported in real-time are correlated on the fly.

With the advent of SKA, providing fantastic sensitivity and intermediate resolution at low frequencies, there will have to be a focus in the long term on the use of Global baselines and frequencies of $5 \mathrm{GHz}$ and higher. A more flexibly organised global VLBI array must be realized that can be deployed in tailor-made configurations for the best science. 


\section{JIVE developments}

\subsection{Research and Development}

In view of these EVN ambitions, first a next-generation correlator must provide more capacity for processing these higher bandwidths, but also more flexibility for spectral line science, astrometry, pulsar binning and many-bit processing. Although yet only a modest number of experiments exceed the parameters offered by the old MkIV correlator, the processing is now predominantly carried out on the EVN software correlator at JIVE (SFXC), which implements a more accurate correlation algorithm in a more flexible architecture [18]. The SFXC platform already offers a number of additional new scientific capabilities, including pulsar binning, wide field imaging and high spectral resolution modes. In particular it is very powerful for near-field correlation to measure spacecraft signals.

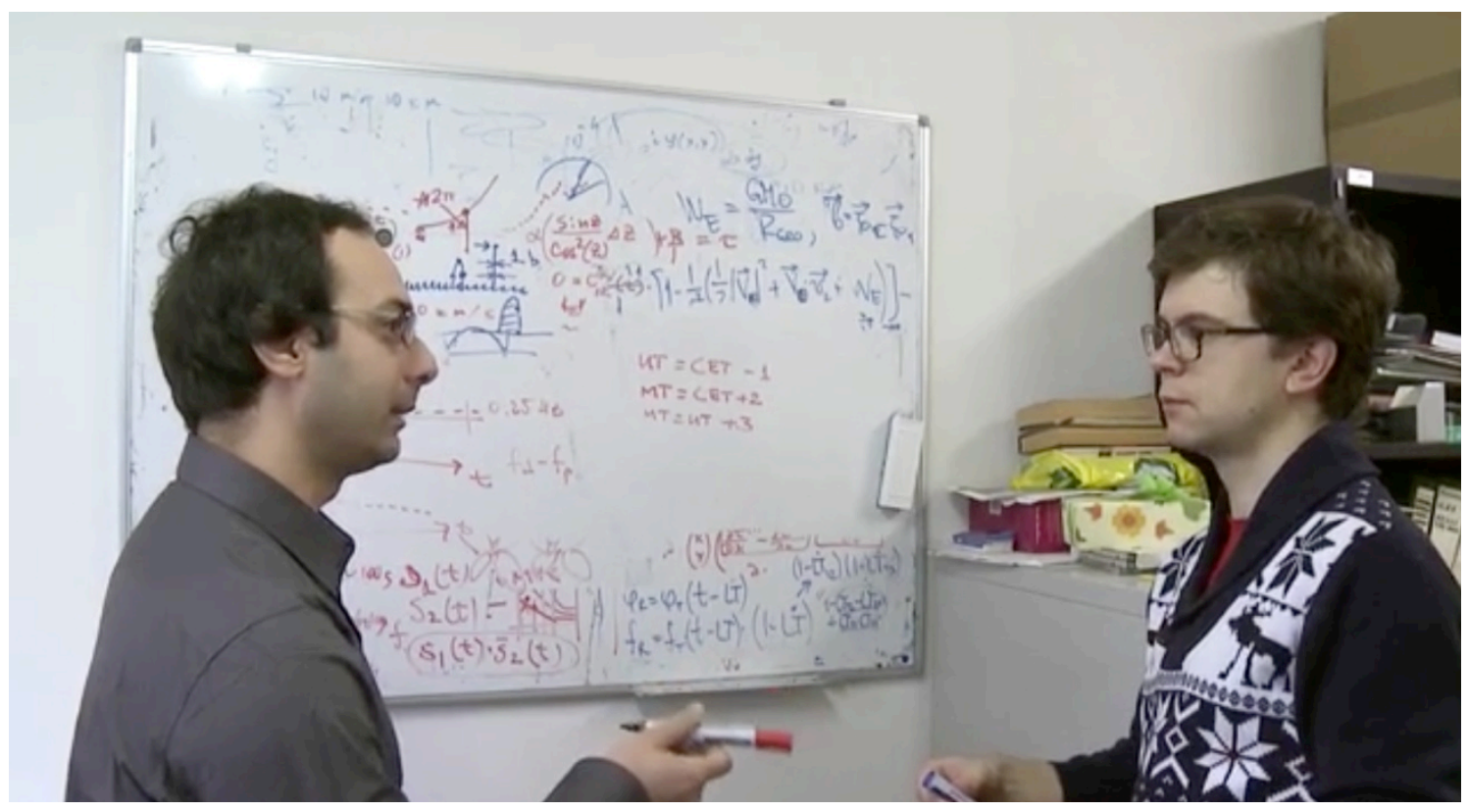

Figure 1. JIVE staff discussing details of the Space application programme; clip from the JIVE movie available at http://www.jive.nl/jive-movie-released

It is questioned whether a software correlator, running on standard computing hardware, is the solution for the EVN on the longer term, especially in view of the development of energy costs. Looking at the EVN (and global) ambitions to have more stations and the desire for increasing bandwidths, one can anticipate the need to process 32 stations at 4 or maybe even 16 Gbps per second. Such a correlator would need to be a hundred times more powerful than the current data processor. Like some of the (other) SKA pathfinders, JIVE is actively pursuing for correlation on FPGA based architectures [19].

At JIVE the NEXPReS project is coordinated, aiming at implementing the e-VLBI enhancements. The focus is on transparent, high bandwidth caching mechanisms and bandwidth-on-demand protocols. With these in place, it should be possible to reserve guaranteed, high-capacity connections for VLBI experiments and release them back for other applications afterwards. Also the distributed correlator effort focuses on allocating resources 
flexibly. Using computing resources at the VLBI telescope sites, it should be possible to farm out the correlation needs for a modest VLBI network deployed for transient event follow-up.

The future use also dictates that the existing VLBI arrays become much more flexible in scheduling and operations. Operational models must evolve in such a way that users can easily define the optimal array in spatial coverage, frequency and sensitivity for their observations, even on short notice. It should be possible to flexibly schedule and adapt observations in order to reach a guaranteed level of quality, taking for example atmospheric conditions into account.

\subsection{JIVE governance}

JIVE implements its mission to support VLBI for the EVN by a range of activities. The main component is the correlation of the dominant fraction of EVN and Global experiments. With this comes the task of user support, from documenting and maintaining the user interfaces to the facility and its data, to in-person help in all stages of the process. There is also a network support aspect implemented at JIVE, monitoring the data quality and calibration factors of all the EVN elements. In addition, a large number of JIVE staff is involved in the aforementioned R\&D programmes. Because over the years these have been implemented mostly as EC-funded projects, considerable expertise has been built up for running complex, multi-partner projects following a variety of funding schemes. Notably, JIVE has taken a central administrative role in three consecutive RadioNet projects.

JIVE is registered as a Dutch not-for-profit foundation with its staff employed at the Dutch organization for scientific research, NWO. The financial contributions are organised through a 5 year MOU and are committed by 10 organization in 9 countries, amongst which South Africa's NRF features for the first time in 2012. Because 2012 is the last year of the current 5-year cycle, preparations started to renew the MOU for JIVE. This process started with a formal review of JIVE and how it implements its mission. A delegation of international experts visited JIVE in March 2012 and reviewed its achievements and plans for the future. In its final report, published at http://www.jive.nl/jive-review-2012, the review panel had especially high praise for JIVE's support role and R\&D activities, concerning digital technology, connectivity, space science applications and astronomical software, as well as the science output from JIVE staff. The panel also recommended making the power of VLBI better known across the astronomy community, stating, "There is almost no area of astronomy which does not benefit from VLBI observations".

In the strategy that the review panel endorsed, JIVE plans to transform its legal base into a so-called European Research Infrastructure Consortium (ERIC). This supposedly provides a more fitting base for the international collaboration in which countries take responsibility for JIVE, rather than the personal involvement that is implied in the foundation platform. Moreover, there are fiscal advantages with the new structure. Finally, one may hope that the ERIC will be rooted more firmly in the European science arena, fitting with JIVE's desire to take on more responsibilities in European radio astronomy.

During 2012 the partners in JIVE were formulating their commitments for the continuity of the JIVE services and its careful transition to a new legal entity. Despite the difficult economic climate for public funding in most European countries, the JIVE partners were able to formulate a strong commitment to sustain the JIVE activities and secure its role in the governance of European radio astronomy. 


\section{References}

[1] D. Fenech, High-resolution monitoring of SNR in M82, PoS(11th EVN Symposium)003

[2] S. Frey, SDSS J1425+3231: a suspected dual radio quasar as seen with the EVN, PoS (11th EVN Symposium)066

[3] M. Gawronski, Project RISARD, PoS(11th EVN Symposium)043

[4] M. Giroletti, e-EVN observations of the first gamma-ray nova V407 Cyg, PoS(11th EVN Symposium) 047

[5] C. Goddi, L. Moscadelli and A. Sanna, Detailed structures of accretion and outflow probed by molecular masers in high-mass protostars, PoS(11th EVN Symposium)031

[6] L. Gurvits, Planetary Radio Interferometry and Doppler Experiment (PRIDE): a multidisciplinary enhancement of space science missions, PoS(11th EVN Symposium)029

[7] Y. Kovalev RadioAstron space VLBI mission: current status and early results of AGN studies, PoS(11th EVN Symposium)012

[8] R. Laing, VLBI science with ALMA, PoS(11th EVN Symposium)052

[9] I. Marti-Vidal, J.M. Marcaide, A. Alberdi and A. Brunthaler, Jet precession in the AGN of M81. Physical parameters and evidence of a binary black hole, PoS(11th EVN Symposium)065

[10]E. Middelberg, The AGN component of the faint radio source population, $\mathrm{PoS}(11$ th EVN Symposium)017

[11]J. Moldon, M. Ribó and J.M Paredes, Periodic radio morphology of the gamma-ray binaries, PoS(11th EVN Symposium)048

[12] G. Molera Calves, VLBI tracking of the Venus Express spacecraft in the Venus polar upper atmosphere, PoS(11th EVN Symposium)030

[13]T. Muxlow, The European VLBI Network - An introduction and description of recent enhancements and scientific highlights, PoS(11th EVN Symposium)001

[14]M. Perez-Torres, Arp 299: The show must go on, PoS(11th EVN Symposium)002

[15]K. Rygl, A. Brunthaler, A. Sanna, K.M. Menten, M.J. Reid, H.J. Van Langevelde, M. Honma, K. Torstensson and K. Fujisawa, Parallaxes and proper motions of interstellar masers toward the Cygnus $X$ star-forming complex, PoS(11 th EVN Symposium $) 040$

[16]A. Sanna, The high-mass SFR G23.01-0.41: from the HMC to the VLBI maser kinematics, PoS(11th EVN Symposium)035

[17]G. Surcis, W.H.T. Vlemmings, H.J. Van Langevelde and B. Hutawarakorn Kramer, High resolution magnetic field measurements in high-mass star-forming regions using masers, PoS(11th EVN Symposium)034

[18]A. Szomoru, Recent developments of the SFXC software correlator, PoS(11th EVN Symposium) 110

[19]A. Szomoru, The UniBoard, PoS(11th EVN Symposium)111

[20] R. Vermeulen, LOFAR status and first astronomical results, PoS(11th EVN Symposium)069 